\title{
Integral electric current characteristics of unsteady-state processes of current passage through liquid dielectrics
}

\author{
V. A. Chirkov, Yu. K. Stishkov, A. A. Sitnikov \\ Physics Department \\ St. Petersburg State University \\ 198504, St. Petersburg, Russia
}

\begin{abstract}
The paper presents the technique for computing current-time characteristics of the transient regime, taking place after the pulsed application or change of high voltage, and current-voltage ones obtained with the voltage sawtooth modulation. The basis of the simulation is the complete set of electrohydrodynamic equations with both the convective and the migration mechanisms of charge transport being taken into account jointly. The numerical calculations were performed using commercial software package COMSOL Multiphysics based on the finite element method. The simulation was carried out for the needle-plane electrode system. The unsteady-state current passage processes, including electrohydrodynamic flow formation, were calculated. The interrelation between the latter and the total current passing through the electrical circuit were investigated. The shape and features of the current-voltage characteristics obtained with the voltage sawtooth modulation were shown to be dependent on the mechanism of charge formation. The qualitative comparison between the simulated and the original experimental data has shown a good agreement.
\end{abstract}

Index Terms - EHD flow; computer simulation; conductivity; injection; fieldenhanced dissociation.

\section{INTRODUCTION}

The current passage through dielectric liquids is an important, yet complicated process. The total current passing through a cell with low-conducting liquid is caused by the combination of ion formation and charge transport processes that are strongly dependent on the electric field strength [1]. Besides, liquid properties, cell geometry and electrode material also affect the total current. Therefore, on the one hand, the integral electric current characteristics (in particular, current-voltage and current-time ones) are of great interest since they contain valuable information on the processes taking place inside the bulk. But on the other hand, the interpretation of the corresponding results is very complicated due to superposition a number of factors. The latter, in turn, leads in practice to using but one quantity, lowvoltage conductivity (or, in rare instances, current-voltage characteristic), as the parameter characterizing the physical state of the system with liquid dielectric.

In view of the foregoing, the computer simulation of the integral electric current characteristics is a topical question since it lets one investigate the shapes of current-voltage and current-time characteristics (CVC and CTC, respectively) and interrelate their features with processes taking place inside the bulk. The corresponding issue has been studied insufficiently due to a number of complications that have hindered numerical calculations earlier and led to using simplified approaches, e.g. one-dimensional problem statement and the approximation of migration-ohmic charge transport [2]. Recently, however, a new method for computer simulation of current passage processes was proposed owing to the development of programs for numerical calculation of partial differential equations and considerable growth of computing power. The method enables one to make allowance for the convective charge transport (that can be of importance in the electric conductivity of dielectric liquids [3]), consider both injection and dissociation mechanisms of ion formation [4], and take into account a finite level of lowvoltage conductivity [5]. Besides, the method was supplemented with the technique for calculation of the total current passing through the external electrical circuit in the unsteady-state regime [6]. Thus, whereas a number of computer investigations of high-voltage current passage processes deal with hydrostatic case (e.g., [2, 7, 8]), the present work features joint computation of the electrohydrodynamic flow and the integral electric-current 
characteristic, which, in turn, allows one to advance in the interpretation of the experimental data and verify existing theoretical models.

The paper presents the technique for computing CTC of the transient regime, taking place after the pulsed application or change of high voltage, and CVC obtained with the voltage sawtooth modulation. The former type of data is a widespread one while the use of the latter type is quite limited. Its application for the investigation of high-voltage conductivity of a dielectric liquid was suggested in [9], where the corresponding sets of data were called the dynamical currentvoltage characteristics or DCVCs. The main advantage of the method is the rapidity of the experimental data acquisition in a wide voltage range. Thus, the whole measurement process takes place under constant external conditions. Then, using the DCVC method makes it possible to verify the reproducibility and stability over time of the characterization as opposed to the classical method where the same verification is very time-consuming. At last, the rapidity of data acquisition reduces the probability of accidental current fluctuations caused by mechanical impurities (dust), since the latter usually fail to get in the near-electrode area during a short voltage modulation period. Along with the advantages for experimental use, application of DCVC is also promising even for the computer simulation. Above all, its computing requires less time than simulating classical $\mathrm{CVC}$ where obtaining steady-state currents is necessary for every voltage value. Besides, dynamical CVCs contain complementary information that is thoroughly analyzed in the present work. In spite of a number of advantages, the application of DCVC has been restricted until now since it requires allowing for the capacitive current that distorts the experimental oscillograms and since there were no computer models that could explain the totality of experimental data.

\section{MATHEMATICAL MODEL AND SIMULATION TECHNIQUE}

Computer simulation is carried out for the needle-plane electrode system with highly non-uniform electric field distribution. In such a system, the local electric field strength in near-electrode area is many times its average, which enables studying high-voltage processes of charge formation even at relatively low voltage across the interelectrode gap to avoid the electrical breakdown of liquid [10].

The simulation base is the complete set of electrohydrodynamic (EHD) equations [9], describing the current passage processes in isothermal incompressible liquid dielectrics:

$$
\begin{gathered}
\operatorname{div}(\boldsymbol{E})=\rho / \varepsilon \varepsilon_{0} \\
\boldsymbol{E}=-\nabla \varphi \\
\partial n_{i} / \partial t+\operatorname{div}\left(\boldsymbol{j}_{i}\right)=g(n, \boldsymbol{E}) \\
\boldsymbol{j}_{i}=n_{i} b_{i} \boldsymbol{E}-D_{i} \nabla n_{i}+n_{i} \boldsymbol{u} \\
\rho=e\left(n_{1}-n_{2}\right)
\end{gathered}
$$

$$
\begin{gathered}
\gamma \partial \boldsymbol{u} / \partial t+\gamma(\boldsymbol{u}, \nabla) \boldsymbol{u}=-\nabla P+\eta \Delta \boldsymbol{u}+\rho \boldsymbol{E} \\
\operatorname{div}(\boldsymbol{u})=0,
\end{gathered}
$$

where $\boldsymbol{E}$ is the electric field strength, $\rho$ is the space charge density, $\varphi$ is the electric potential, $n$ is the ion concentration, $\boldsymbol{j}$ is the density of ion flux, $\boldsymbol{u}$ is the fluid velocity, $P$ is the pressure, $\varepsilon$ is the relative electric permittivity, $\gamma$ is the mass density, $\eta$ is the dynamic viscosity, $b$ is the ion mobility, $D$ is the diffusion coefficient, $\varepsilon_{0}$ is the electric constant, $e$ is the elementary electric charge, $t$ is the time; subscript $i$ indicates the ion species. Ions are assumed to be monovalent.

The mechanism of charge formation is taken into account in simulation directly. In the case of the unipolar injection into non-conducting liquid, the source function $g$ in (3) is set to zero. Functional dependence of the injection current density on local electric field strength $j_{\mathrm{inj}, i}(E)$ and condition of charge loss $j_{\text {loss, } i}$ is set at the surface of every electrode:

$$
\begin{gathered}
j_{\text {inj, }, i}(E)=f_{i}(E) \\
j_{\text {loss }, i}(E)=d_{i}(n, E) .
\end{gathered}
$$

The theoretical determination of functional dependence $f_{i}(E)$ is a very intricate issue that requires taking into account kinetic reactions occurring at the metal-liquid interface. At present, even very complicated theories [11] dealing with electrochemical reactions at the electrode surface fail to explain all variety of the observable results. Moreover, the formulation of universal boundary conditions for the charge transport equation is impossible since various physicochemical processes can occur under high voltage depending on the properties of electrode surface and liquid [12].

In spite of complexity of near-electrode reactions, introducing a phenomenological function dependence of injection on the local electric field strength lets one advance in a simulation and obtain satisfactory results. Thus, we use the dependence of the Fowler-Nordheim equation kind, where coefficients $A, B$ were found from the experimental data on the current-voltage characteristic [4] and were slightly increased $\left(A=3.3 \cdot 10^{17} 1 / \mathrm{m}^{2}, \quad B=3.4 \cdot 10^{7} \mathrm{~V} / \mathrm{m}\right)$ to change the injection intensity in the simulation:

$$
f_{i}(E)=A \exp (-B / E)
$$

Function $d_{i}(n, E)$ describing the charge loss is typically set proportional to the ion concentration in the near-electrode area. One of the alternatives of the loss condition is to set function $d_{i}(n, E)$ equal to the total current density for ions arriving to the boundary from the bulk:

$$
d_{i}(n, E)=n_{i} b_{i} E_{N}-D_{i} \nabla_{N} n_{i},
$$

where subscript $N$ denotes the normal to the surface of the electrode. 
When the low-voltage conductivity is taken into account, the source function is set as [13]:

$$
\begin{gathered}
g(n, \boldsymbol{E})=\sigma_{0}^{2} /\left(e\left(b_{1}+b_{2}\right) \varepsilon \varepsilon_{0}\right) F(p)- \\
e\left(b_{1}+b_{2}\right) /\left(\varepsilon \varepsilon_{0}\right) n_{1} n_{2},
\end{gathered}
$$

where $\sigma_{0}$ is the low-voltage conductivity and $F$ is the relative increase in the dissociation rate. $F$ is assumed to be 1 except the last case considered in the paper.

The numerical computations of the set of equations (1)-(7) are performed using software COMSOL Multiphysics ${ }^{\circledR}$ based on the finite element method. The simulation technique is described in detail in $[6,14]$, where one can find more information about its features. A 2D axisymmetric model was created in simulation. The geometry of computer model and boundary conditions for the set of equations are presented in Figure 1.

The liquid properties correspond to those of transformer oil: $\varepsilon=2.2, \gamma=870 \mathrm{~kg} / \mathrm{m}^{3}, \eta=0.025 \mathrm{~Pa} \cdot \mathrm{s},|b|=10^{-8} \mathrm{~m}^{2} / \mathrm{V} \cdot \mathrm{s}$ (which is assumed to be the same for ions of both polarities). The diffusion coefficient of monovalent ions according to the Einstein relation is $D=2.5 \cdot 10^{-10} \mathrm{~m}^{2} / \mathrm{s}$; however, inflated value $D=10^{-7} \mathrm{~m}^{2} / \mathrm{s}$ is used in the simulation for better convergence, which nevertheless does not affect the results of computations [13]. Low-voltage conductivity $\sigma_{0}$ is a parameter of study, and its value is varied with other properties of the liquid left unchanged. This paper considers the conductivity values in the range from 0 to $10^{-9} \mathrm{~S} / \mathrm{m}$.

There are several ways to calculate the total value of the electric current passing through the external electrical circuit, but the most convenient and reliable one in the unsteady-state regime is the approach based on the Shockley-Ramo theorem [15-17]. According to the latter, the total current flowing through the external electric circuit is given by:

$$
I=\int_{V}\left(\boldsymbol{j}^{*}, \boldsymbol{f}_{E}\right) d \boldsymbol{x},
$$

where $j^{*}$ is the total electric current density, $f_{E}$ is the weighting electric field (in units of inverse distance) [17], $V$ is the cell volume, and $\boldsymbol{x}$ is the spatial coordinate.

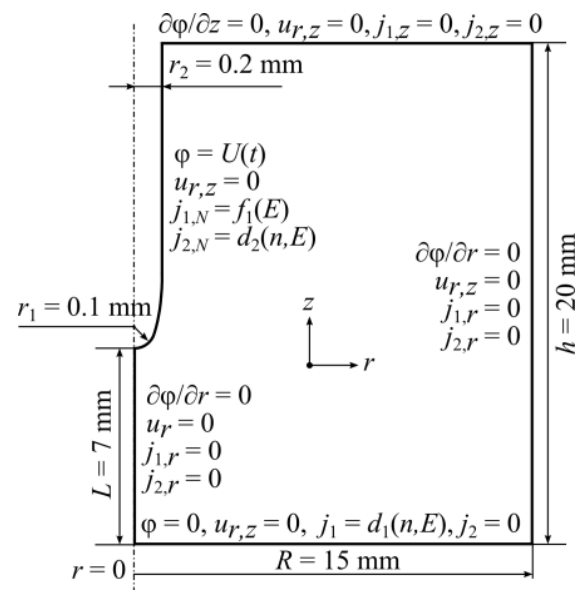

Figure 1. Geometry of computer model and boundary conditions for the set of equations.
If the voltage across the gap varies, the capacitive current will run and the specific additional term has to be included in (13):

$$
I=\int_{V}\left(\boldsymbol{j}^{*}, \boldsymbol{f}_{E}\right) d \boldsymbol{x}+\varepsilon \varepsilon_{0} \partial \varphi / \partial t \int_{V}\left(\boldsymbol{f}_{E}, \boldsymbol{f}_{E}\right) d \boldsymbol{x}
$$

\section{RESULTS AND DISCUSSION}

\subsection{SIMULATION OF CURRENT-TIME CHARACTERISTICS}

To begin with, consider the integral electric current characteristics (or so-called "charging current") in the case of the pulsed application of high voltage $\left(U(t)=U_{0}\right.$ at $\left.t \geq 0\right)$. First of all, analyze the corresponding processes in the case of unipolar injection into non-conducting liquid (i.e., at $\sigma_{0}=0$ ) and at voltage $7 \mathrm{kV}$ (that corresponds to the average electric field strength $10 \mathrm{kV} / \mathrm{cm}$ ). Figure $2 \mathrm{a}$ presents several consecutive snap-shots of space charge density distribution during the transient regime. A charged layer emerges around the needle electrode in initially non-conducting liquid after the voltage is turned on. Ions start to migrate in the electric field with electrical current emerging in the circuit (the beginning of the curve 1 in Figure $2 b$ ). After that, the quantity of the injected ions rises and EHD flow emerges. The latter accelerates the process of ion propagation to the counter electrode, which ensures steep increase in the total current.
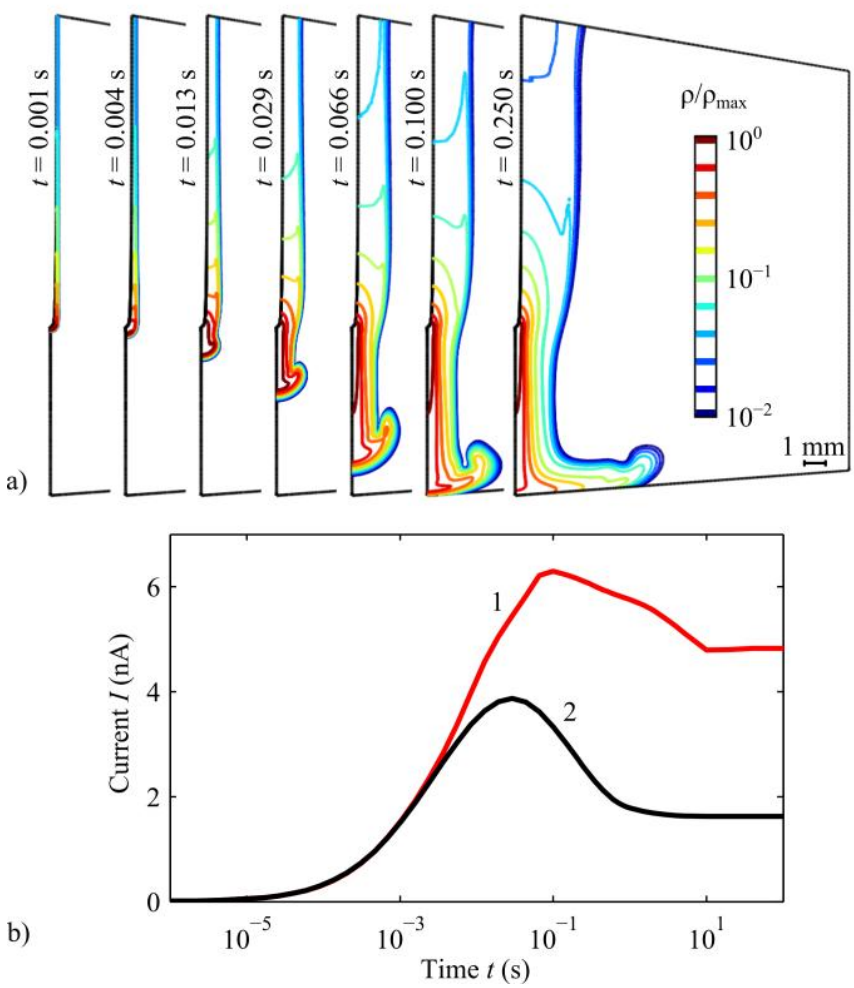

Figure 2. Contour plots of space charge density distribution on logarithmic scale at consecutive instants of time (a) and current-time characteristics of the transient process in the model of the injection into nonconducting liquid ((b), 1-EHD and 2-hydrostatic cases); $\rho_{\max }=$ $0.06 \mathrm{C} / \mathrm{m}^{3}$. 
The maximum value of the calculated CTC corresponds to the instant when the charged jet reaches the counter electrode. The characteristic time interval for the corresponding process amounts to $0.1 \mathrm{~s}$. Then, although the area of space charge localization slightly increases due to spreading of ions along the plane electrode, the total current begins to decrease since the space charge reduces the electric field strength near the active electrode and, thereby, lessens the injection intensity. The total current undergoes no changes after $10 \mathrm{~s}$ when the space charge reaches its steady-state distribution. Thus, in the considered model of charge injection into non-conducting liquid, two specific time spans can be distinguished: the time of charged-jet propagation to the counter electrode and that of space-charge accumulation in the bulk.

To underlie the significance of taking into consideration convective mechanism of charge transport, consider "charging current" obtained in a simplified model when EHD flow is disregarded, i.e. in the hydrostatic case (curve 2 in Figure $2 b$ ). On the one hand, the shape of CTC is similar to that obtained in the complete approach. However, on the other hand, the specific time spans of attaining the maximum and steady-state electric current values as well as the values themselves changed. It means that convective charge transport affects both time of adjustment to the external conditions and total conductivity of the system. Therefore, one can conclude that EHD flows are to be computed when investigating unsteady-state integral electric current characteristics in a cell with dielectric liquid, at least, at negligible level of low-voltage conductivity of the latter. Accordingly, all the data presented below were computed with allowance for the convective charge transport.

Proceed to the case of the unipolar injection into liquid with finite (non-zero) conductivity and with the injection function left unchanged. An example of the corresponding CTC (at $\sigma_{0}=10^{-10} \mathrm{~S} / \mathrm{m}$ ) is presented in Figure 3 (curve 1) and has at least two distinctions from the one obtained in the pure injection model. Firstly, the total current starts to grow from the non-zero level since dissociation-recombination reactions provide initial ion concentration at the instant of the voltage turn-on. Secondly, the CTC shows no decrease after the attainment of the maximum since the presence of conductivity hastens the process of getting the steady-state

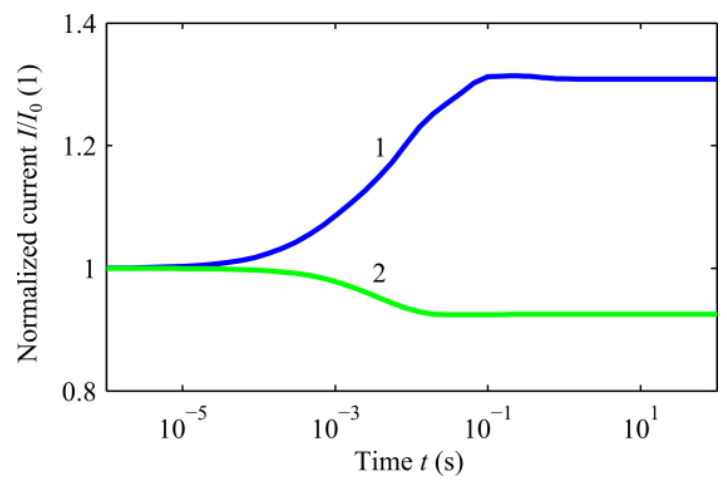

Figure 3. Current-time characteristics of the transient process in the model of the injection into liquid with a finite conductivity $\left(1-\sigma_{0}=\right.$ $10^{-10} \mathrm{~S} / \mathrm{m}$ and $\left.2-\sigma_{0}=10^{-9} \mathrm{~S} / \mathrm{m}\right)$. space charge distribution. The corresponding time scale is the Maxwell relaxation time $\left(\tau_{\mathrm{M}}=\varepsilon \varepsilon_{0} / \sigma_{0}\right)$ that amounts to $0.2 \mathrm{~s}$ at the chosen low-voltage conductivity level. If the liquid conductivity is so high that the conduction current density prevails over the injection one, the Maxwell relaxation time controls the process of the formation of near-electrode dissociation-recombination layers. In these circumstances, EHD flow fails to emerge and the total current passing through the cell decreases from the moment of voltage application (curve 2 in Figure 3, $\sigma_{0}=10^{-9} \mathrm{~S} / \mathrm{m}$ ). In the case, the only specific time span is the Maxwell relaxation one.

\subsection{SIMULATION OF DYNAMICAL CURRENT- VOLTAGE CHARACTERISTICS}

Though the CTC is more common and understandable than DCVC, it has one important shortcoming. Its experimental implementation demands getting voltage-step with rising edge shorter than the specific time span of the CTC (e.g., EHD flow formation). The latter condition is actually impracticable, which leads to the contradictions in the data obtained by different research groups, with the corresponding issue being discussed in [11]. Besides, the sharp voltage increase has to result in the huge capacitive current passing through the external electrical circuit and distorting the CTC. Therefore, the DCVC has a number of advantages from the experimental standpoint and its implementation softens the requirements to the equipment since there is no need to generate voltage-step with a steep rising edge. At the same time, the DCVC as well as CTC lets one investigate transient processes and obtain greater amount of data than the classical (steady-state) CVC.

As above, begin with the case of unipolar injection into non-conducting liquid. To provide clear-cut analysis, we used linearly changing voltage with a single polarity rather than $\mathrm{AC}$ voltage that was used, e.g., in the experiments presented in [18]; nevertheless, the proposed simulation technique is entirely applicable for the AC case. Figure 4 presents the original time dependences of the applied voltage and measured current. The maximum voltage value is chosen to be as high as possible but below the breakdown one. The current starts to rise with a delay since ions emerge in the bulk only after the activation of the injection, i.e. after the

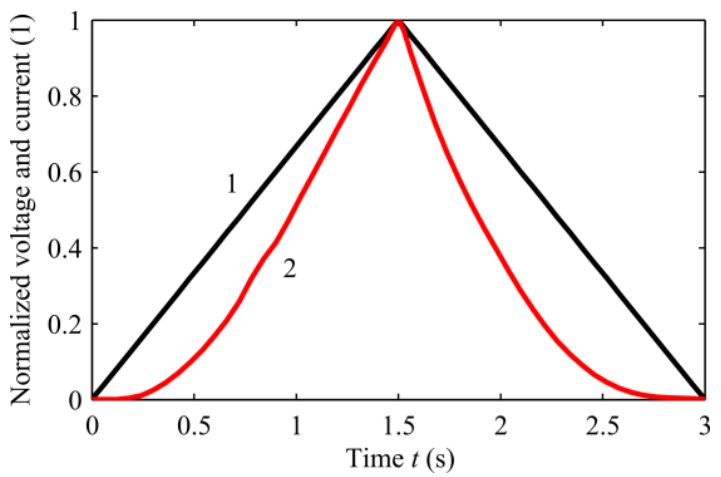

Figure 4. An example of time dependences of the applied voltage (1) and measured current (2) in the case of unipolar injection into non-conducting liquid at $20 \mathrm{kV} / \mathrm{s}$ voltage modulation rate $\left(U_{\max }=30 \mathrm{kV}, I_{\max }=145 \mathrm{nA}\right)$. 
threshold value of the electric field strength. Whereas the total current in the computer simulation is free of the capacitive component (unlike an actual experimental data, e.g., presented in [19]), the data presented in Figure 4 can be used directly for plotting DCVC itself. The corresponding results are presented in Figure 5a for two different rates of voltage modulation ( 20 and $2 \mathrm{kV} / \mathrm{s}$ ) along with the electric current values obtained in the steady-state regime (i.e., steady-state CVC). According to the data presented above, the DCVC in the pure injection model (without dissociation) begins to rise only after the activation of the injection and thereby failing to describe the low-voltage part of CVC. Further, the increasing voltage section is higher than the decreasing voltage one, especially at a high voltage modulation rate. The corresponding difference is the so-called hysteresis. It emerges for the reason that the typical time span of the voltage change is less than that of ion crossing the interelectrode gap, and the system fails to adjust to the changing conditions. The feature is discussed in more detail in [20]. The hysteresis lessens with decreasing the voltage modulation rate, and dynamic CVC almost turns into the steady-state one.

As it was shown above, the conductivity reduces the specific time span of the current steadying. Consequently, dissociation-recombination processes in the bulk are also expected to affect the hysteresis. Figure $5 \mathrm{~b}$ presents the corresponding DCVCs calculated with the injection function left unchanged and without disregarding the low-voltage conductivity. The obtained plots show the hysteresis to reduce at $\sigma_{0}=10^{-10} \mathrm{~S} / \mathrm{m}$ and vanish at $\sigma_{0}=3 \cdot 10^{-10} \mathrm{~S} / \mathrm{m}$ at the same
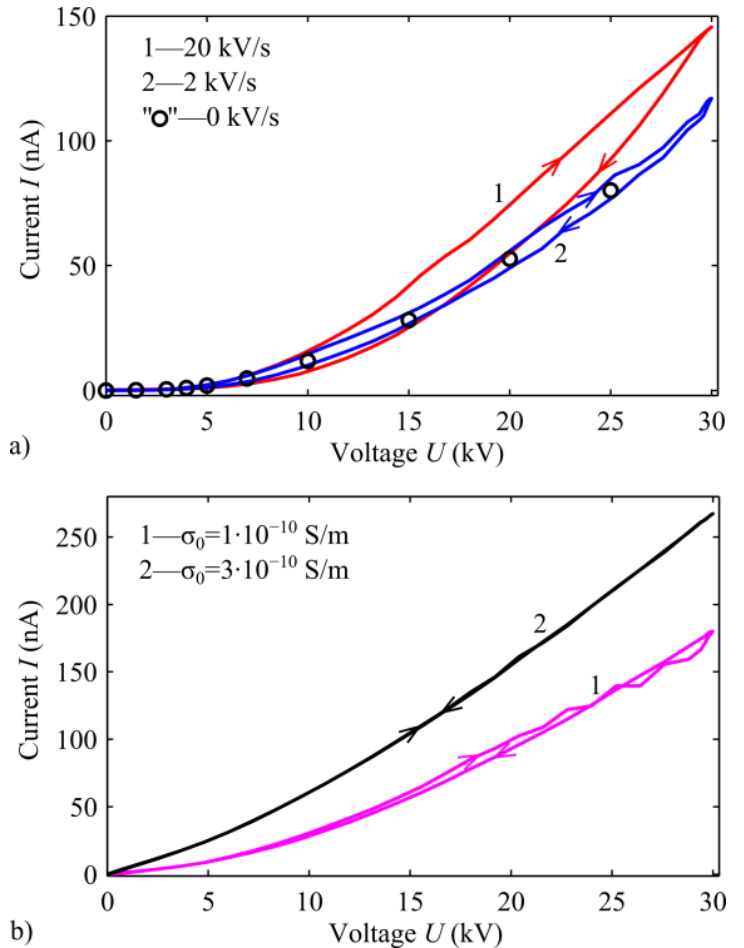

Figure 5. DCVCs in the case of the injection into non-conducting liquid at different voltage modulation rates (a) and those calculated without ignoring the low-voltage conductivity at $20 \mathrm{kV} / \mathrm{s}$ voltage modulation rate (b). voltage modulation rate, though the injected space charge still decreases the electric field strength in the vicinity of the needle tip.

A sharp increase in the dissociation rate is known to take place under the effect of the strong electric field when the strength of the latter exceeds $10^{7} \mathrm{~V} / \mathrm{m}$. Such enhancement is often called the Wien effect, and some researchers believe the effect to underlie the space charge formation in the lowconducting liquids and lead to the EHD flow emergence. To ensure the investigation of the features of high-voltage current passage in the case of the Wien effect, our computer model was supplemented with the explicit dependence of the dissociation rate on the electric field strength. According to [21], the latter is expressed via the Bessel function:

$$
\begin{gathered}
F(p)=I_{1}(4 p) /(2 p) \\
p=e^{2} /\left(2 k_{B} T\right) \sqrt{ }\left(E /\left(4 \pi \varepsilon \varepsilon_{0} e\right)\right),
\end{gathered}
$$

where $I_{1}$ is the modified Bessel function of the first kind, $k_{B}$ is the Boltzmann constant, and $T$ is the temperature.

To simplify the analysis, the injection charge formation was excluded from consideration. However, it is to be emphasized that the obtained DCVCs were calculated without ignoring the convective charge transport, which is of great importance according to the results presented above. The corresponding integral electric current characteristics are shown in Figure 6 a for the voltage modulation rate $20 \mathrm{kV} / \mathrm{s}$ and the following values of the low-voltage conductivity: $\sigma_{0}=$ $10^{-11}, 10^{-10}$ and $10^{-9} \mathrm{~S} / \mathrm{m}$. Since the current level grows considerably with the increasing low-voltage conductivity (from $10^{-11}$ to $10^{-9} \mathrm{~S} / \mathrm{m}$ ), the DCVCs are normalized to the current value that would be obtained at $U=30 \mathrm{kV}$ in the case of constant conductivity. Thus, if the value of the normalized current is 7 (at $30 \mathrm{kV}$ ), it means that the integral conductivity of the cell grows due to the Wien effect sevenfold.

The calculated DCVCs are linear in a relatively wide voltage range (approximately up to $15 \mathrm{kV}$ ), while further voltage increase leads to a steeply rising current. And the main feature of the results is the absence of a hysteresis. The latter is correct both at $\sigma_{0}=10^{-11} \mathrm{~S} / \mathrm{m}$, when Maxwell relaxation time exceeds one second, and at $\sigma_{0}=10^{-9} \mathrm{~S} / \mathrm{m}$, when the Wien effect provides rather high space charge density. The absence of the hysteresis can be explained by the features of EHD flow structure in the case of the Wien effect. Figure $6 \mathrm{~b}$ and $6 \mathrm{c}$ presents contour plots of space charge density and fluid velocity. The former plot shows the charge density to be localized within very narrow jet (even when it is plotted on the logarithmic scale) and its major part to be concentrated in the immediate vicinity of the needle tip rather than in the whole interelectrode gap as it is in the case of the injection charge formation. At the same time, the maximum value of the density exceeds $3 \mathrm{C} / \mathrm{m}^{3}$, which provides very high density of the Coulomb force. Consequently, the emerging EHD flows show very high intensity with the maximum of the fluid velocity exceeding $2 \mathrm{~m} / \mathrm{s}$. Thus, these all provide rapid 


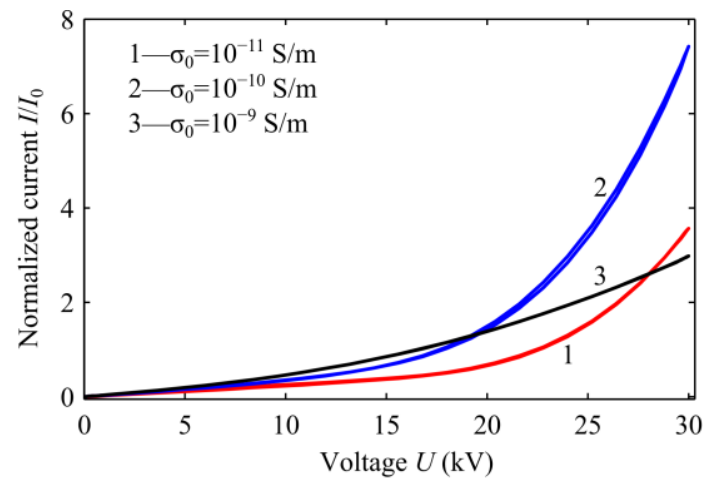

a)
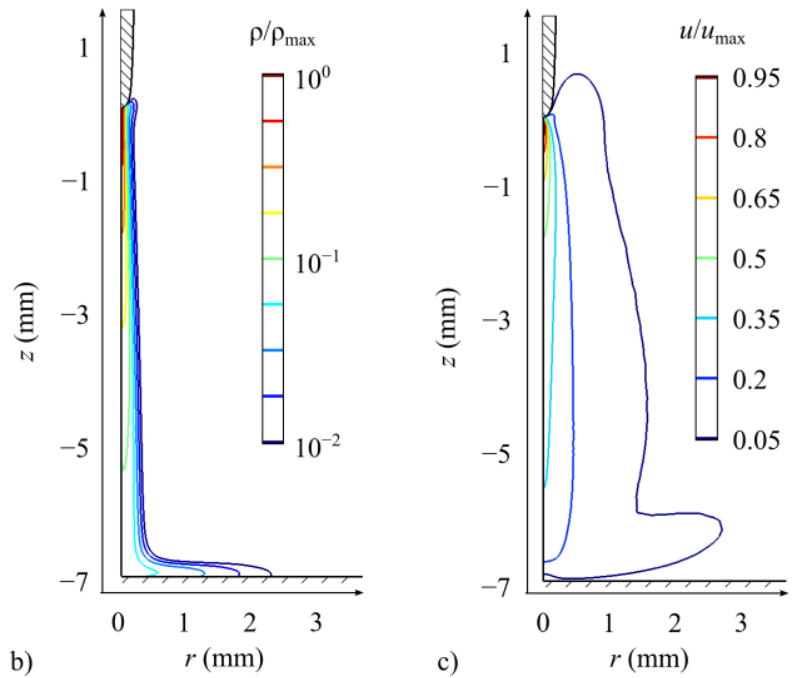

Figure 6. DCVCs (a) and contour plots of space charge density (b) and fluid velocity (c) at $\sigma_{0}=10^{-10} \mathrm{~S} / \mathrm{m}$ and $U=20 \mathrm{kV}$ in the case of fieldenhanced dissociation; $\rho_{\max }=3.2 \mathrm{C} / \mathrm{m}^{3}, u_{\max }=2.1 \mathrm{~m} / \mathrm{s}$.

system adjustment to the changing voltage, at least, in comparison with the case of the injection charge formation.

\subsection{EXPERIMENTAL DYNAMICAL CURRENT- VOLTAGE CHARACTERISTICS}

Consider the original experimental dynamical CVCs (Figure 7) obtained for the needle-plane electrode system with the geometry similar to that used in the simulation. All the original experimental data were processed and the capacitive current was subtracted from the total one. Details of the corresponding experimental technique and additional data can be found in [19]. Figure 7 displays an example of the DCVC of transformer oil with low-voltage conductivity $\sigma_{0}=$ $2 \cdot 10^{-11} \mathrm{~S} / \mathrm{m}$ and at the voltage modulation rate $5 \mathrm{kV} / \mathrm{s}$. At sufficiently low voltage (up to approximately $3 \mathrm{kV}$ ), the dynamical CVC shows linear current growth, which corresponds to the range of the Ohm's law applicability. In the voltage range, the dissociation of the impurity molecules is actually the sole charge formation mechanism while the migration is the main mechanism of ion transport. The section of more rapid current increase is seen at higher voltages, which corresponds to the activation of high-voltage charge formation mechanisms (i.e., the injection or fieldenhanced dissociation) and emergence of EHD flows. At last, the difference between the increasing voltage section and the
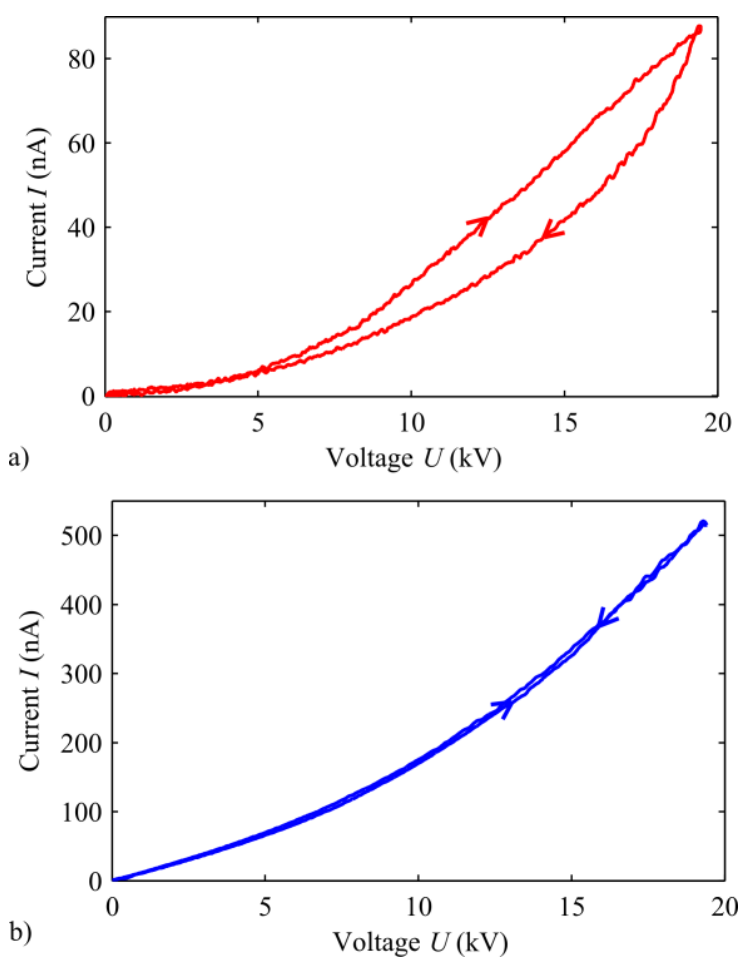

Figure 7. The experimental DCVCs of transformer oil without (a) and with (b) $5 \%$ impurity of butanol at the voltage modulation rate $5 \mathrm{kV} / \mathrm{s}$ for the needle-plane electrode system.

decreasing voltage one (i.e., the hysteresis) is clearly seen in the presented DCVC. However, the hysteresis vanishes (Figure 7b) with the increase in conductivity of the working liquid (up to $2.5 \cdot 10^{-10} \mathrm{~S} / \mathrm{m}$ ) by introduction of an electronacceptor impurity (5\% of butanol) into the transformer oil. Thus, both considered cases are in a good qualitative agreement with the simulated results: the former DCVC corresponds to the simulated case of the injection into liquid with minor conductivity, while the latter - to the case of the field-enhanced dissociation or injection into low-conducting liquid.

\section{CONCLUSION}

The described computer simulation technique lets one calculate the integral electric current characteristics of a cell with dielectric liquid in unsteady-state regime, considering both injection and dissociation mechanisms of ion formation and taking into account the convective charge transport as well as a finite level of the low-voltage conductivity. In particular, the technique is applicable to calculation of the socalled dynamical current-voltage characteristics. In turn, the latter were shown to be opportune for describing the current passage processes inside a cell with dielectric liquid since the corresponding approach has a number of advantages (in particular, low time expenditures and the convenience of the experimental realization) as compared to the classical CVC and CTC. Besides, the DCVC can be used to clarify one of the main issues in EHD about the prevailing charge formation mechanism (that underlies the emergence of space charge in the bulk) since the latter effects on the shape and features of 
the former. Thus, if the injection mechanism of charge formation prevails and if the specific time span of the voltage changing is less than that of the space charge adjustment, the DCVC exhibits hysteresis. Moreover, the less the low-voltage conductivity, the more noticeable the difference between the two parts of DCVC corresponding to increasing and decreasing voltage. However, there is no hysteresis in the model of field-enhanced dissociation even at relatively small low-voltage conductivity of the working liquid. At last, the features of DCVC revealed in the simulation are observable in the actual experiments and are in a good qualitative agreement with them.

\section{ACKNOWLEDGMENT}

The reported study was supported by RFBR, research project No. 14-08-31507 mol_a. Research was carried out using computer resources provided by Resource Center "Computer Center of SPbU" (http://cc.spbu.ru).

\section{REFERENCES}

[1] A. Denat, "Conduction and breakdown initiation in dielectric liquids", IEEE Intern. Conf. Dielectr. Liquids (ICDL), Trondheim, Norway, 2011.

[2] J. Jadidian, J. G. Hwang, M. Zahn, N. Lavesson, O. Widlund, and K. Borg "Migration-Ohmic Charge Transport in Liquid-Solid Insulation Systems", IEEE Intern. Conf. Dielectr. Liquids (ICDL), Trondheim, Norway, 2011.

[3] V.A. Chirkov, Yu.K. Stishkov, "Electrohydrodynamic mechanism of the electric conduction", Intern. Sympos. Electrohydrodynamics (ISEHD), Gdansk, Poland, 2012.

[4] Yu. Stishkov and V. Chirkov, "Dependence of the Electrohydrodynamic Flows Structure in Very Non-Uniform Electric Field on the Charge Formation Mechanism", IEEE Intern. Conf. Dielectr. Liquids (ICDL), Trondheim, Norway, 2011.

[5] I.A. Ashikhmin, Yu.K. Stishkov, "Influence of the Level of the LowVoltage Conduction on the Structure of the Through Electrohydrodynamic Flow in a Symmetric Electrode System", Surf. Eng. and Appl. Electrochem., vol. 50, no. 3, pp. 246-252, 2014.

[6] V.A. Chirkov, Yu.K. Stishkov, "Current-time characteristic of the transient regime of electrohydrodynamic flow formation", J. Electrostat., vol.71, pp. 484-488, 2013.

[7] N. Lavesson, L. Walfridsson, O. Hjortstam and J. Schiessling, "Modelling and Measurement of Field Dependent Resistivity of Transformer Oil", IEEE Intern. Conf. Dielectr. Liquids (ICDL), Bled, Slovenia, 2014.

[8] F. Vahidi, S. Tenbohlen, M. Rösner, C. Perrier, and H. Fink, "Space Charge Formation in Insulating Liquids under DC Stresses", IEEE Intern. Conf. Dielectr. Liquids (ICDL), Bled, Slovenia, 2014.

[9] Yu.K. Stishkov, A.A. Ostapenko, Electrohydrodynamical Flows in Liquid Dielectrics (in Russian), Leningr. Gos. Univ., Leningrad, 1989.

[10] Y. Sha, Y. Zhou, D. Nie, Z. Wu, and J. Deng, "A Study on Electric Conduction of Transformer Oil", IEEE Trans. Dielectr. Insul., Vol. 21, No. 3, pp. 1061-1070, 2014.

[11] A.I. Zhakin, "Near-electrode and transient processes in liquid dielectrics", Phys. Usp., vol. 49, pp. 275-295, 2006.

[12] A.I. Zhakin, "Electrohydrodynamics", Phys. Usp., vol. 55, pp. 465-488, 2012.

[13] Yu.K. Stishkov, V.A. Chirkov, "Formation of Electrohydrodynamic Flows in Strongly Nonuniform Electric Fields for Two Charge-Formation Modes”, Tech. Phys., vol. 57, no. 1, pp. 1-11, 2012.
[14] Yu. K. Stishkov and V. A. Chirkov, "Structure of the Near-Electrode Dissociation-Recombination Charged Layers at Various Low-Voltage Conductivities of a Low-Conducting Liquid", Tech. Phys., vol. 58, no. 12, pp. 1822-1830, 2013.

[15] A. Shockley, "Currents to conductors induced by a moving point charge", J. Appl. Phys., vol. 9, pp. 635-636, 1938.

[16] S. Ramo, "Currents induced by electron motion", Proc. Inst. Radio Eng., vol. 27, pp. 584-585, 1939.

[17] L.-A. Hamel, M. Julien, “Generalized demonstration of Ramo's theorem with space charge and polarization effects", Nucl. Instrum. and Methods in Phys. Res. A, vol. 597, pp. 207-211, 2008.

[18] T. Grav and L. E. Lundgaard, "Currents in AC Stressed Liquid Insulated Needle Plane Gap", IEEE Intern. Conf. Dielectr. Liquids (ICDL), Bled, Slovenia, 2014.

[19] V. A. Chirkov, Yu. K. Stishkov, and A. A. Sitnikov, "Features of current passage processes in liquid dielectrics at the injection and dissociation mechanisms of charge formation", Intern. Sympos. Electrohydrodynamics (ISEHD), Okinawa, Japan, 2014.

[20] Yu.K. Stishkov, V.A. Chirkov, and A.A. Sitnikov, "Dynamic CurrentVoltage Characteristics of Weakly Conducting Liquids in Highly NonUniform Electric Field", Surf. Eng. and Appl. Electrochem., vol. 50, no. 2, pp. 135-140, 2014.

[21] L. Onsager, "Deviations from Ohm's law in weak electrolytes", J. Chem. Phys., vol. 2, pp. 599-615, 1934.

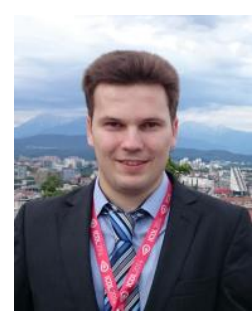

Vladimir Chirkov was born in Leningrad, USSR in 1986. He received the B.Sc. and M.Sc. degrees in applied mathematics and physics from St. Petersburg State University, St. Petersburg, Russia in 2007 and 2009, respectively. In 2014, he was awarded the Ph.D. degree at St. Petersburg State University for his research into high-voltage processes of the current passage through dielectric liquids in a wide voltage range. His research interests include electrohydrodynamics, mechanisms of charge formation, gas discharges, and the computer simulation of the corresponding processes. He is a member of IEEE.

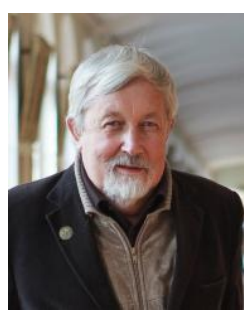

Yury Stishkov was born in Tbilisi, USSR in 1941. He received his M.Sc. degree in physics from Tbilisi State University in 1964, the Ph.D. and D.Sc. degrees in physics from Leningrad State University in 1971 and 1986, respectively. In 2002 he became full professor at the St. Petersburg State University, Russia. He is the co-chairman of the International Conference on Modern Problems of Electrophysics and Electrohydrodynamics of Liquids and member of editorial board of the Surface Engineering and Applied Electrochemistry journal. He has worked in the areas of electrohydrodynamics, magnetohydrodynamics, mechanisms of charge formation, ionic conductivity, heat transfer enhancement, and plasma physics. He is currently Professor and Head of Electrophysics Research and Education Center.

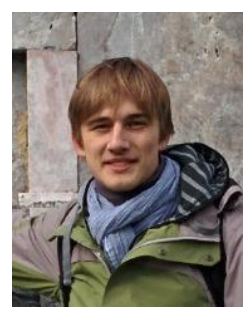

Andrei Sitnikov was born in Nizhny Tagil, USSR in 1991. He received the B.Sc. degree in applied mathematics and physics from St. Petersburg State University, St. Petersburg, Russia in 2013. His research interest concerns electrohydrodynamics, mechanisms of charge formation, and especially experimental testing techniques. 